\title{
On the evolution equation for the rest stress in rate-dependent plasticity
}

\author{
V.A. Lubarda*, D.J. Benson \\ Department of Mechanical and Aerospace Engineering, University of California, \\ San Diego, La Jolla, CA 92093-0411, USA
}

Received in final revised form 24 October 2000

\begin{abstract}
Three different structures of the evolution equation for the rest stress are derived. They correspond to different models of viscoplastic response, constructed by three-dimensional generalization of simple one-dimensional rheological models. Isotropic, kinematic and combined isotropic-kinematic hardening, with an evolution equation for the back stress, are incorporated in the constitutive framework. The results may be of interest in the analytical and numerical studies of the rate-dependent inelastic response. (C) 2002 Elsevier Science Ltd. All rights reserved.
\end{abstract}

Keywords: B. Elastic-viscoplastic material; Rheological models; Rest stress; Back stress

\section{Introduction}

There are two types of constitutive equations used for the rate-dependent plastic response of metals and alloys. In one approach, there is no yield surface in the model and plastic deformation commences from the onset of loading, although it may be exceedingly small below certain levels of applied stress. This type of modeling is particularly advocated by researchers in materials science, who view inelastic deformation process as inherently time-dependent. For example, this view is supported by the dislocation dynamics study of crystallographic slip in metals, e.g. Johnston and Gilman (1959). Since there is no separation of time-independent and creep effects, the modeling is often referred to as the unified creep-plasticity theory

\footnotetext{
* Corresponding author. Tel.: + 1-858-534-3169; fax: + 1-858-534-5698.
}

E-mail address: vlubarda@ucsd.edu (V.A. Lubarda). 
(Hart, 1970; Krieg, 1977; Miller, 1987). In his analysis of rate-dependent behavior of metals, Rice $(1970,1971)$ showed that plastic rate of deformation can be derived from a scalar flow potential $\Omega$ as its gradient

$$
\mathbf{D}^{p}=\frac{\partial \Omega(\boldsymbol{\sigma}, \theta, \mathcal{H})}{\partial \boldsymbol{\sigma}}
$$

provided that the rate of shearing on any given slip system within a crystalline grain depends on local stresses only through the resolved shear stress. The history of deformation is represented by the pattern of internal rearrangements $\mathcal{H}$, and the absolute temperature is $\theta$. Geometrically, the plastic part of the rate of deformation is normal to surfaces of constant flow potential in stress space. Time-independent behavior can be recovered under certain idealizations-neglecting creep and rate effects - as an appropriate limit.

The power-law representation of the flow potential has been often utilized,

$$
\Omega=\frac{2 \dot{\gamma}^{0}}{m+1}\left(J_{2}^{1 / 2} / k\right)^{m} J_{2}^{1 / 2}, \quad J_{2}=\frac{1}{2} \boldsymbol{\sigma}^{\prime}: \boldsymbol{\sigma}^{\prime}
$$

where $k=k(\theta, \vartheta)$ is the reference shear stress, dependent on the temperature and equivalent plastic strain

$$
\vartheta=\int_{0}^{t}\left(2 \mathbf{D}^{p}: \mathbf{D}^{p}\right)^{1 / 2} d t .
$$

The trace product is denoted by : and $\dot{\gamma}^{0}$ is the reference shear strain rate. The material rate-sensitivity parameter is $m$. The corresponding plastic part of the rate of deformation is from Eq. (1),

$$
\mathbf{D}^{p}=\dot{\gamma}^{0}\left(J_{2}^{1 / 2} / k\right)^{m} \frac{\boldsymbol{\sigma}^{\prime}}{J_{2}^{1 / 2}} .
$$

Similar expressions can be obtained with other choices of the flow potential function, such as those corresponding to Bodner and Partom (1975), Ponter and Leckie (1976), or Johnson and Cook (1983) model. Since there is no yield surface and loading/unloading criteria in unified creep-plasticity theory, some authors refer to these constitutive models as nonlinearly viscoelastic models. However, by using an appropriate large value of the parameter $m$, the rate-dependent model is able to reproduce almost rate-independent behavior. The function $x^{m}$ can in that sense be viewed as the regularizing function ( $x$ stands for $J_{2}^{1 / 2} / k$ ). Other examples of regularizing functions include $\tanh (x / m)$ and $[\exp (x)-1]^{m}$.

Another type of modeling of rate-dependent inelastic response is frequently employed in high strain-rate dynamic problems. This is referred to as viscoplastic modeling (Sokolovskii, 1948; Malvern, 1951; Perzyna, 1963, 1966). There are two surfaces in the model. The dynamic loading surface carries the current stress state 
and encloses the static yield surface associated with quasi-static loading. The plastic rate of deformation is codirectional with the outer normal to the loading surface. Its magnitude is proportional to a scalar overstress, defined by the positive difference between the measures of dynamic and static states of stress. For example, if the static yield surface is defined by the von Mises condition

$$
f=\frac{1}{2} \boldsymbol{\beta}: \boldsymbol{\beta}-k^{2}(\vartheta)=0
$$

the dynamic loading surface is

$$
\hat{f}=\frac{1}{2} \sigma^{\prime}: \sigma^{\prime}-\hat{k}^{2}=0,
$$

The deviatoric stress tensor in quasi-static loading, at a given level of generalized plastic strain $\vartheta$, is $\boldsymbol{\beta}$, which is referred to as the rest stress. The scalar overstress measure is defined by

$$
J_{2}^{1 / 2}-k(\vartheta), \quad J_{2}^{1 / 2}=\left(\frac{1}{2} \boldsymbol{\sigma}^{\prime}: \boldsymbol{\sigma}^{\prime}\right)^{1 / 2}
$$

The plastic rate of deformation is then

$$
\mathbf{D}^{p}=\frac{1}{\eta}\left[1-\frac{k(\vartheta)}{J_{2}^{1 / 2}}\right] \frac{\partial \hat{f}}{\partial \boldsymbol{\sigma}}=\frac{1}{\eta}\left[1-\frac{k(\vartheta)}{J_{2}^{1 / 2}}\right] \boldsymbol{\sigma}^{\prime}, \quad 1-\frac{k(\vartheta)}{J_{2}^{1 / 2}}>0
$$

where $\eta$ is the viscosity coefficient. In a quasi-static test, the viscosity coefficient is equal to zero, while $J_{2}^{1 / 2}=k(\vartheta)$.

In an alternative approach, introduce the static yield surface $f(\boldsymbol{\beta})=0$, and define the plastic rate of deformation as

$$
\mathbf{D}^{p}=\frac{1}{\eta}\left(\boldsymbol{\sigma}^{\prime}-\boldsymbol{\beta}\right)
$$

This applies when $f(\boldsymbol{\sigma})>0$, so that the current stress state $\sigma$ is outside the static yield surface $f(\boldsymbol{\beta})=0$. The constitutive formulation can then proceed by constructing an evolution or growth equation for the rest stress $\boldsymbol{\beta}$, and an appropriate expression for the viscosity parameter $\eta$. In general, it is not required that $\boldsymbol{\sigma}^{\prime}$ and $\boldsymbol{\beta}$ are coaxial tensors, although for some models or for some loading paths they may be. The rest stress considered in this paper is related to equilibrium stress of Krempl's (1996) model, where there is no static yield surface and the equilibrium stress grows from the zero initial value (see also Krempl and Gleason, 1996). The objective of this paper is a derivation of the evolution equation for the rest stress. After giving a brief summary of some basic results from the rate-independent plasticity, needed for the constitutive analysis presented in this 
paper, we deduce three different evolution equations for the rest stress. Two of them follow from the constitutive models in which the decomposition of the rate of deformation appears, while in one the stress rate decomposition is used. The utilized constitutive models are obtained by three-dimensional generalization from simple one-dimensional rheological models. This facilitates physical interpretation of the resulting expressions and enables evolution equations to be derived, under certain assumptions, rather than postulated. Isotropic, kinematic and combined isotropickinematic hardening are all encompassed by the presented framework. In the latter two cases, an evolution equation for the back stress is also used to account for anisotropic hardening. Derived results may be of importance in analytical and numerical evaluations of rate-dependent material response, under high strain-rate proportional and non-proportional loading.

\section{Rate-independent plasticity}

The basic constitutive structure of rate-independent plasticity with isotropic and kinematic hardening is reviewed in this section, since these results will be used in the constitutive elaboration of rate-dependent plasticity in subsequent sections. The one-dimensional spring-friction element is shown in Fig. 1. The elastic modulus is $E$. The elastic range is exceeded when applied stress exceeds the current yield stress $\sigma_{Y}$. The total strain rate is the sum of elastic and plastic parts, such that

$$
\dot{\varepsilon}=\left(\frac{1}{E}+\frac{1}{H^{\mathrm{p}}}\right) \dot{\sigma},
$$

where $H^{\mathrm{P}}=\mathrm{d} \sigma / \mathrm{d} \epsilon^{\mathrm{P}}$ is the instantaneous plastic modulus.

A three-dimensional generalization of Eq. (10) is an expression for the rate of deformation tensor $\mathbf{D}$ in terms of instantaneous elastic and plastic compliance tensors and an objective stress rate. For example, we can write

$$
\mathbf{D}=\left(\boldsymbol{M}^{\mathrm{e}}+\boldsymbol{M}^{\mathrm{p}}\right): \stackrel{\circ}{\underline{\sigma}}
$$

where

$$
\underline{\dot{\sigma}}=\stackrel{\circ}{\sigma}+\boldsymbol{\sigma} \operatorname{tr} \mathbf{D}, \stackrel{\circ}{\boldsymbol{\sigma}}=\dot{\boldsymbol{\sigma}}-\mathbf{W} \cdot \boldsymbol{\sigma}+\boldsymbol{\sigma} \cdot \mathbf{W} .
$$

The Cauchy stress tensor is $\boldsymbol{\sigma}$, the material spin tensor is $\mathbf{W}$, the superposed circle designates the Jaumann corotational rate and $\vartheta$ tr stands for the trace. The material

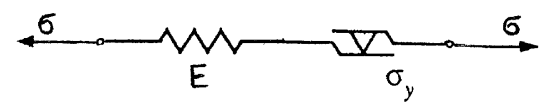

Fig. 1. The spring-friction element simulating one-dimensional elastoplastic response. The elastic modulus is $E$ and the current yield stress is $\sigma_{Y}$. 
time derivative of $\boldsymbol{\sigma}$, on some quasi-static scale, is denoted by $\dot{\boldsymbol{\sigma}}$. The elastic and plastic compliance tensors corresponding to $\underline{\sigma}$ are $\boldsymbol{M}^{\mathrm{e}}$ and $\boldsymbol{M}^{\mathrm{p}}$. For infinitesimally small elastic components of strain, the elastic compliance tensor of an isotropic material is

$$
\boldsymbol{M}^{\mathrm{e}}=\frac{1}{2 \mu} \boldsymbol{J}+\frac{1}{3 \kappa} \boldsymbol{K}
$$

where $\mu$ and $\kappa$ are the elastic shear and bulk moduli. The base tensors $\boldsymbol{J}$ and $\boldsymbol{K}$ sum to give the fourth-order unit tensor, $\boldsymbol{J}+\boldsymbol{K}=\boldsymbol{I}$. The rectangular components of $\boldsymbol{I}$ and $\boldsymbol{K}$ are

$$
I_{i j k l}=\frac{1}{2}\left(\delta_{i k} \delta_{j l}+\delta_{i l} \delta_{j k}\right), \quad K_{i j k l}=\frac{1}{3} \delta_{i j} \delta_{k l} .
$$

The components of the second-order unit tensor are the Kronecker deltas $\delta_{i j}$.

\subsection{Isotropic hardening}

If plasticity is governed by isotropic hardening and an associative flow rule with a von Mises yield condition

$$
\frac{1}{2} \sigma^{\prime}: \sigma^{\prime}-k^{2}(\vartheta)
$$

where $k(\vartheta)$ is the yield stress in simple shear, the instantaneous plastic compliance tensor is

$$
\boldsymbol{M}^{\mathrm{p}}=\frac{1}{2 h^{\mathrm{p}}} \frac{\boldsymbol{\sigma}^{\prime} \otimes \boldsymbol{\sigma}^{\prime}}{\boldsymbol{\sigma}^{\prime}: \boldsymbol{\sigma}^{\prime}}
$$

The plastic modulus in a simple shear test is $h^{\mathrm{p}}=\mathrm{d} k / \mathrm{d} \vartheta$, and $\otimes$ stands for the tensor outer product (the rectangular components of $\boldsymbol{\sigma}^{\prime} \otimes \boldsymbol{\sigma}^{\prime}$ being $\sigma_{i j}^{\prime} \sigma_{k l}^{\prime}$ ). The equivalent plastic strain is defined by Eq. (3). The elastoplastic constitutive structure is consequently

$$
\mathbf{D}=\left(\frac{1}{2 \mu} \boldsymbol{J}+\frac{1}{3 \kappa} \boldsymbol{K}+\frac{1}{2 h^{\mathrm{p}}} \frac{\boldsymbol{\sigma}^{\prime} \otimes \boldsymbol{\sigma}^{\prime}}{\boldsymbol{\sigma}^{\prime}: \boldsymbol{\sigma}^{\prime}}\right): \underline{\stackrel{\circ}{\boldsymbol{\sigma}}} .
$$

If a material is in the hardening range $\left(h^{\mathrm{p}}>0\right)$, the plastic loading condition is $\boldsymbol{\sigma}^{\prime}: \underline{\circ}>0$. The inverted constitutive structure, expressing the stress rate in terms of the rate of deformation, is

$$
\stackrel{\circ}{\boldsymbol{\sigma}}=\left(2 \mu \boldsymbol{J}+3 \kappa \boldsymbol{K}-\frac{2 \mu}{1+h^{\mathrm{p}} / \mu} \frac{\boldsymbol{\sigma}^{\prime} \otimes \boldsymbol{\sigma}^{\prime}}{\boldsymbol{\sigma}^{\prime}: \boldsymbol{\sigma}^{\prime}}\right): \mathbf{D} .
$$


The corresponding plastic loading condition is $\boldsymbol{\sigma}^{\prime}: \mathbf{D}>0$.

It is noted that the plastic rate of deformation can be expressed either in terms of the stress rate or the total rate of deformation, as

$$
\mathbf{D}^{p}=\frac{1}{2 h^{\mathrm{p}}} \frac{\boldsymbol{\sigma}^{\prime} \otimes \boldsymbol{\sigma}^{\prime}}{\boldsymbol{\sigma}^{\prime}: \boldsymbol{\sigma}^{\prime}}: \underline{\stackrel{o}{\boldsymbol{\sigma}}}=\frac{1}{1+h^{\mathrm{p}} / \mu} \frac{\boldsymbol{\sigma}^{\prime} \otimes \boldsymbol{\sigma}^{\prime}}{\boldsymbol{\sigma}^{\prime}: \boldsymbol{\sigma}^{\prime}}: \mathbf{D} .
$$

In performing the inverse operation in Eq. (17), the following result may be conveniently used. The fourth-order tensor

$$
\boldsymbol{H}=a \boldsymbol{J}+b \boldsymbol{K}+c \frac{\boldsymbol{\sigma}^{\prime} \otimes \boldsymbol{\sigma}^{\prime}}{\boldsymbol{\sigma}^{\prime}: \boldsymbol{\sigma}^{\prime}},
$$

where $a, b$ and $c$ are scalar parameters and $\boldsymbol{\sigma}^{\prime}$ is a deviatoric second-order tensor, has the inverse

$$
\boldsymbol{H}^{-1}=\frac{1}{a} \boldsymbol{J}+\frac{1}{b} \boldsymbol{K}-\frac{c}{a(a+c)} \frac{\boldsymbol{\sigma}^{\prime} \otimes \boldsymbol{\sigma}^{\prime}}{\boldsymbol{\sigma}^{\prime}: \boldsymbol{\sigma}^{\prime}}
$$

This can be easily verified by noting that $\boldsymbol{J}: \boldsymbol{J}=\boldsymbol{J}$ and $\boldsymbol{K}: \boldsymbol{K}=\boldsymbol{K}$, as well as $\boldsymbol{J}$ : $\boldsymbol{K}=\boldsymbol{K}: \boldsymbol{J}=\mathbf{0}$. In the trace operation with any second-order tensor $\boldsymbol{A}$, the tensor $\boldsymbol{J}$ extracts its deviatoric part, while the tensor $\boldsymbol{K}$ extracts its spherical part $\left(\boldsymbol{J}: \boldsymbol{A}=\boldsymbol{A}^{\prime}\right.$ and $\boldsymbol{K}: \boldsymbol{A}=\boldsymbol{A}-\boldsymbol{A}^{\prime}$ ). Thus, $\boldsymbol{J}: \boldsymbol{\sigma}^{\prime}=\boldsymbol{\sigma}^{\prime}$ and $\boldsymbol{K}: \boldsymbol{\sigma}^{\prime}=\mathbf{0}$. The tensors $\boldsymbol{J}$ and $\boldsymbol{K}$ have been frequently employed in the constitutive studies (e.g. Hill, 1965). Note that a deviatoric tensor $\mathbf{N}=\boldsymbol{\sigma}^{\prime} /\left\|\boldsymbol{\sigma}^{\prime}\right\|$ has only four independent components, since $\|\mathbf{N}\|=(\mathbf{N}: \mathbf{N})^{1 / 2}=1$ (e.g. van Houtte, 1994). The fourth-order tensor $\mathbf{N} \otimes \mathbf{N}$ in general has fourteen linearly independent components.

\subsection{Kinematic hardening}

According to the linear kinematic hardening model, the yield surface in stress space translates in the direction of plastic rate of deformation. At an arbitrary instant of deformation the center of the yield surface is at the stress point $\boldsymbol{\alpha}$, such that

$$
\frac{1}{2}\left(\sigma^{\prime}-\alpha\right):\left(\sigma^{\prime}-\alpha\right)=k_{0}^{2}
$$

The constant radius of the yield surface is $k_{0}$, the initial yield stress in simple shear. The deviatoric stress $\boldsymbol{\alpha}$ is referred to as the back stress. The evolution equation for this stress is, according to Prager's (1956) linear kinematic hardening model,

$$
\underline{\alpha}=2 h^{\mathrm{p}} \mathbf{D}^{\mathrm{p}}, \quad \underline{\stackrel{\circ}{\alpha}}=\stackrel{\circ}{\boldsymbol{\alpha}}+\boldsymbol{\alpha} t r \mathbf{D} .
$$

The ordinary Jaumann derivative is used to make the evolution equation objective. Other derivatives were also considered in the literature (e.g. Lee et al., 1983). The instantaneous plastic compliance tensor is consequently 


$$
\boldsymbol{M}^{\mathrm{p}}=\frac{1}{2 h^{\mathrm{p}}} \frac{\left(\boldsymbol{\sigma}^{\prime}-\boldsymbol{\alpha}\right) \otimes\left(\boldsymbol{\sigma}^{\prime}-\boldsymbol{\alpha}\right)}{\left(\boldsymbol{\sigma}^{\prime}-\boldsymbol{\alpha}\right):\left(\boldsymbol{\sigma}^{\prime}-\boldsymbol{\alpha}\right)} .
$$

This gives rise to the elastoplastic constitutive structure

$$
\mathbf{D}=\left[\frac{1}{2 \mu} \boldsymbol{J}+\frac{1}{3 \kappa} \boldsymbol{K}+\frac{1}{2 h^{\mathrm{p}}} \frac{\left(\boldsymbol{\sigma}^{\prime}-\boldsymbol{\alpha}\right) \otimes\left(\boldsymbol{\sigma}^{\prime}-\boldsymbol{\alpha}\right)}{\left(\boldsymbol{\sigma}^{\prime}-\boldsymbol{\alpha}\right):\left(\boldsymbol{\sigma}^{\prime}-\boldsymbol{\alpha}\right)}\right]: \underline{\stackrel{\circ}{\sigma}} .
$$

The plastic loading condition in the hardening range is $\left(\boldsymbol{\sigma}^{\prime}-\boldsymbol{\alpha}\right): \underline{\stackrel{\sigma}{\sigma}}>0$. The inverted form is

$$
\underline{\circ}=\left[2 \mu \boldsymbol{J}+3 \kappa \boldsymbol{K}-\frac{2 \mu}{1+h^{\mathrm{p}} / \mu} \frac{\left(\boldsymbol{\sigma}^{\prime}-\boldsymbol{\alpha}\right) \otimes\left(\boldsymbol{\sigma}^{\prime}-\boldsymbol{\alpha}\right)}{\left(\boldsymbol{\sigma}^{\prime}-\boldsymbol{\alpha}\right):\left(\boldsymbol{\sigma}^{\prime}-\boldsymbol{\alpha}\right)}\right]: \mathbf{D},
$$

provided that $\left(\boldsymbol{\sigma}^{\prime}-\boldsymbol{\alpha}\right): \mathbf{D}>0$.

The evolution equation (23) can be expressed in terms of the stress rate, or the rate of deformation, as

$$
\begin{aligned}
& \stackrel{\circ}{\underline{\alpha}}=\frac{\left(\boldsymbol{\sigma}^{\prime}-\boldsymbol{\alpha}\right) \otimes\left(\boldsymbol{\sigma}^{\prime}-\boldsymbol{\alpha}\right)}{\left(\boldsymbol{\sigma}^{\prime}-\boldsymbol{\alpha}\right):\left(\boldsymbol{\sigma}^{\prime}-\boldsymbol{\alpha}\right)}: \stackrel{\circ}{\boldsymbol{\sigma}}, \quad\left(\boldsymbol{\sigma}^{\prime}-\boldsymbol{\alpha}\right): \stackrel{\circ}{\underline{\boldsymbol{\sigma}}}>0, \\
& \stackrel{\circ}{\alpha}=\frac{2 h^{\mathrm{p}}}{1+h^{\mathrm{p}} / \mu} \frac{\left(\boldsymbol{\sigma}^{\prime}-\boldsymbol{\alpha}\right) \otimes\left(\boldsymbol{\sigma}^{\prime}-\boldsymbol{\alpha}\right)}{\left(\boldsymbol{\sigma}^{\prime}-\boldsymbol{\alpha}\right):\left(\boldsymbol{\sigma}^{\prime}-\boldsymbol{\alpha}\right)}: \mathbf{D}, \quad\left(\boldsymbol{\sigma}^{\prime}-\boldsymbol{\alpha}\right): \mathbf{D}>0 .
\end{aligned}
$$

A nonlinear kinematic hardening model of Armstrong and Frederick (1966) is obtained if, instead of Eq. (23), the evolution equation for $\boldsymbol{\alpha}$ is taken to be

$$
\underline{\alpha}=2 h \mathbf{D}^{\mathrm{p}}+c \boldsymbol{\alpha}\left(\mathbf{D}^{\mathrm{p}}: \mathbf{D}^{\mathrm{p}}\right)^{1 / 2}
$$

where $h>0$ and $c<0$ are appropriate material parameters. This expresses $\stackrel{\circ}{\boldsymbol{\alpha}}$ as a homogeneous function of degree one in the components of $\mathbf{D}^{\mathrm{p}}$. The added nonlinear term in Eq. (29) is sometimes referred to as a recall term (Chaboche, 1989). It follows that

$$
\mathbf{D}^{\mathrm{p}}=\frac{1}{2 h(1+m)} \frac{\left(\boldsymbol{\sigma}^{\prime}-\boldsymbol{\alpha}\right) \otimes\left(\boldsymbol{\sigma}^{\prime}-\boldsymbol{\alpha}\right)}{\left(\boldsymbol{\sigma}^{\prime}-\boldsymbol{\alpha}\right):\left(\boldsymbol{\sigma}^{\prime}-\boldsymbol{\alpha}\right)}: \stackrel{\stackrel{\circ}{\boldsymbol{\sigma}}}{ },
$$

where

$$
m=\frac{c}{2 h} \frac{\left(\boldsymbol{\sigma}^{\prime}-\boldsymbol{\alpha}\right): \boldsymbol{\alpha}}{\left[\left(\boldsymbol{\sigma}^{\prime}-\boldsymbol{\alpha}\right):\left(\boldsymbol{\sigma}^{\prime}-\boldsymbol{\alpha}\right)\right]^{1 / 2}} .
$$

In modeling cyclic plasticity it may be convenient to additively decompose $\boldsymbol{\alpha}$ into two or more constituents, and construct separate evolution equation for each of these (e.g. Moosbrugger and McDowell, 1989). Such an approach has also been 
employed in viscoplastic modeling which incorporates the concept of back stress. See also Lubarda et al. (1993).

\subsection{Combined isotropic-nonlinear kinematic hardening}

In the case of combined isotropic-kinematic hardening, the yield surface translates and expands, such that

$$
\frac{1}{2}\left(\boldsymbol{\sigma}^{\prime}-\boldsymbol{\alpha}\right):\left(\boldsymbol{\sigma}^{\prime}-\boldsymbol{\alpha}\right)=k_{\boldsymbol{\alpha}}^{2}(\vartheta)
$$

where $\boldsymbol{\alpha}$ represents its current center and $k_{\alpha}(\vartheta)$ its current radius. With the nonlinear evolution equation for the back stress given by Eq. (29), we obtain

$$
\mathbf{D}^{\mathrm{p}}=\frac{1}{2 h_{\boldsymbol{\alpha}}^{\mathrm{p}}+2 h(1+m)} \frac{\left(\boldsymbol{\sigma}^{\prime}-\boldsymbol{\alpha}\right) \otimes\left(\boldsymbol{\sigma}^{\prime}-\boldsymbol{\alpha}\right)}{\left(\boldsymbol{\sigma}^{\prime}-\boldsymbol{\alpha}\right):\left(\boldsymbol{\sigma}^{\prime}-\boldsymbol{\alpha}\right)}: \underline{\stackrel{\sigma}{\boldsymbol{\sigma}}} .
$$

The rate of the yield surface expansion is specified by $h_{\alpha}^{\mathrm{p}}=\mathrm{d} k_{\boldsymbol{\alpha}} / \mathrm{d} \vartheta$, and $m$ is defined by Eq. (31). This encompasses isotropic and kinematic hardening models. In the former case $h_{\alpha}^{\mathrm{p}}=h^{\mathrm{p}}$ (plastic tangent modulus in simple shear), while in the latter case $h_{\alpha}^{\mathrm{p}}=0$.

\section{Viscoplasticity}

The simplest viscoplasticity model is based on the spring-dashpot-friction element shown in Fig. 2. The elastic modulus is $E$, the viscosity coefficient is $\eta$, and the yield stress is $\sigma_{\mathrm{Y}}$. The total strain rate is the sum of the elastic part $\dot{\epsilon}^{\mathrm{e}}=\dot{\sigma} / E$, and the plastic part

$$
\dot{\epsilon}^{\mathrm{p}}=\frac{1}{\eta}\left(\sigma-\sigma_{Y}\right), \quad \sigma>\sigma_{Y} .
$$

The stress difference $\sigma-\sigma_{Y}>0$ is the portion of applied stress carried by the dashpot, usually referred to as the overstress. The strain rates in the dashpot element and parallel friction element are equal to each other, so that

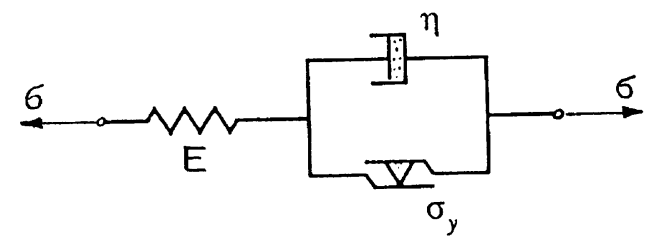

Fig. 2. One-dimensional viscoplastic model. A dashpot with viscosity $\eta$ is connected in parallel to a friction element with the current yield stress $\sigma_{Y}$. The elastcic modulus of the spring element is $E$. 


$$
\frac{1}{H^{\mathrm{p}}} \dot{\sigma}_{Y}=\frac{1}{\eta}\left(\sigma-\sigma_{Y}\right), \quad \sigma>\sigma_{Y}
$$

where $H^{\mathrm{p}}=\mathrm{d} \sigma / \mathrm{d} \epsilon^{\mathrm{p}}$ is the instantaneous plastic modulus. For $\sigma<\sigma_{Y}$, only elastic deformation takes place and $\dot{\epsilon}=\dot{\sigma} / E$.

A three-dimensional generalization of Eq. (34) is obtained by introducing the static yield surface $f(\boldsymbol{\beta})=0$ for the rest stress $\boldsymbol{\beta}$, and by defining the plastic rate of deformation as

$$
\mathbf{D}^{P}=\frac{1}{\eta}\left(\boldsymbol{\sigma}^{\prime}-\boldsymbol{\beta}\right)
$$

This holds for $f(\sigma)>0$, so that the current stress state is outside the static yield surface. If $f(\boldsymbol{\sigma}) \leqslant 0$, only elastic deformation takes place $\left(\mathbf{D}^{\mathrm{p}}=\mathbf{0}\right)$. The deviatoric rest stress $\boldsymbol{\beta}$ represents the equilibrium stress under quasistatic loading conditions (viscosity $\eta$ is then equal to zero). The stress difference $\boldsymbol{\sigma}^{\prime}-\boldsymbol{\beta}$ is the overstress tensor. The viscosity parameter $\eta$ is, in general, a scalar function of the overstress and other variables introduced in the specific material model. To complete the analysis, a constitutive expression for the rest stress $\boldsymbol{\beta}$ must be provided. This can be accomplished by constructing either an evolution-type equation for the objective rate of the rest stress, or an explicit equation for the rest stress itself. The two approaches are considered below.

\subsection{Evolution equation for the rest stress}

An evolution equation for the rest stress can be constructed with the help of the three-dimensional generalization of Eq. (35). For example, with the isotropic hardening model of Section 2.1 and with the von Mises static yield condition of Eq. (5), we have

$$
\frac{1}{2 h^{\mathrm{p}}} \frac{\boldsymbol{\beta} \otimes \boldsymbol{\beta}}{\boldsymbol{\beta}: \boldsymbol{\beta}}: \stackrel{\circ}{\boldsymbol{\beta}}=\frac{1}{\eta}\left(\boldsymbol{\sigma}^{\prime}-\boldsymbol{\beta}\right) .
$$

This shows that $\mathbf{D}^{\mathrm{p}}$ is codirectional with $\partial f / \partial \boldsymbol{\beta}=\boldsymbol{\beta}$, and that $\boldsymbol{\beta}$ and $\boldsymbol{\sigma}^{\prime}-\boldsymbol{\beta}$ are coaxial tensors. The plastic modulus at the equivalent plastic strain, $\vartheta$ is $h^{\mathrm{p}}$. In the hardening range, $\boldsymbol{\beta}: \boldsymbol{\beta}>0$ and $h^{\mathrm{p}}>0$. By taking the trace product of (37) with $\boldsymbol{\beta}$, there follows

$$
\frac{1}{2 h^{p}} \boldsymbol{\beta}: \stackrel{\circ}{\boldsymbol{\beta}}=\frac{1}{\eta} \boldsymbol{\beta}:\left(\boldsymbol{\sigma}^{\prime}-\boldsymbol{\beta}\right) \text {. }
$$

This suggests that the evolution equation for $\boldsymbol{\beta}$ can be represented by

$$
\stackrel{\circ}{\boldsymbol{\beta}}=\frac{2 h^{\mathrm{p}}}{\eta}\left(\boldsymbol{\sigma}^{\prime}-\boldsymbol{\beta}\right)+\mathbf{B},
$$


where $\mathbf{B}$ is the second-order deviatoric tensor orthogonal to the rest stress, i.e., $\mathbf{B}$ : $\boldsymbol{\beta}=0$. In order that the principal directions of $\boldsymbol{\beta}$ remain parallel to those of stress $\boldsymbol{\sigma}^{\prime}$, we define $\mathbf{B}$ to be proportional to the component of stress rate $\dot{\sigma}^{\prime}$ tangent to the static yield surface, thus normal to $\boldsymbol{\beta}$. Such a tensor is

$$
\mathbf{B}=\varphi\left(\underline{\circ}^{\prime}-\frac{\boldsymbol{\sigma}^{\prime} \otimes \boldsymbol{\sigma}^{\prime}}{\boldsymbol{\sigma}^{\prime}: \boldsymbol{\sigma}^{\prime}}: \underline{\circ}\right),
$$

where $\varphi$ is a scaling parameter, given by

$$
\varphi=\left(\frac{\boldsymbol{\beta}: \boldsymbol{\beta}}{\boldsymbol{\sigma}^{\prime}: \boldsymbol{\sigma}^{\prime}}\right)^{1 / 2}
$$

Consequently, the evolution equation for the rest stress becomes

$$
\stackrel{\circ}{\boldsymbol{\beta}}=\frac{2 h^{\mathrm{p}}}{\eta}\left(\boldsymbol{\sigma}^{\prime}-\boldsymbol{\beta}\right)+\varphi\left(\underline{\circ}^{\prime}-\frac{\boldsymbol{\sigma}^{\prime} \otimes \boldsymbol{\sigma}^{\prime}}{\boldsymbol{\sigma}^{\prime}: \boldsymbol{\sigma}^{\prime}}: \underline{\stackrel{\sigma}{\sigma}}\right) .
$$

In the case of kinematic hardening considered in Section 2.2 and the static yield condition defined by

$$
f=\frac{1}{2}(\boldsymbol{\beta}-\boldsymbol{\alpha}):(\boldsymbol{\beta}-\boldsymbol{\alpha})-k_{0}^{2}=0
$$

where $\boldsymbol{\alpha}$ is the current center of the surface $f=0$, we have, in place of Eq. (38),

$$
\frac{1}{2 h^{\mathrm{p}}} \frac{(\boldsymbol{\beta}-\boldsymbol{\alpha}) \otimes(\boldsymbol{\beta}-\boldsymbol{\alpha})}{(\boldsymbol{\beta}-\boldsymbol{\alpha}):(\boldsymbol{\beta}-\boldsymbol{\alpha})}: \stackrel{\circ}{\boldsymbol{\beta}}=\frac{1}{\eta}\left(\boldsymbol{\sigma}^{\prime}-\boldsymbol{\beta}\right) .
$$

The tensors $(\boldsymbol{\beta}-\boldsymbol{\alpha})$ and $\left(\boldsymbol{\sigma}^{\prime}-\boldsymbol{\beta}\right)$ are coaxial, so that $(\boldsymbol{\beta}-\boldsymbol{\alpha}):\left(\boldsymbol{\sigma}^{\prime}-\boldsymbol{\beta}\right)>0$. In the hardening range, the condition $(\boldsymbol{\beta}-\boldsymbol{\alpha}): \stackrel{\boldsymbol{\beta}}{>}>0$ holds. Upon taking the trace product of (44) with $(\boldsymbol{\beta}-\boldsymbol{\alpha})$, there follows

$$
\frac{1}{2 h^{p}}(\boldsymbol{\beta}-\boldsymbol{\alpha}): \stackrel{\stackrel{\boldsymbol{\beta}}{ }}{=}=\frac{1}{\eta}(\boldsymbol{\beta}-\boldsymbol{\alpha}):\left(\boldsymbol{\sigma}^{\prime}-\boldsymbol{\beta}\right) .
$$

This again suggests an evolution equation for the rest stress $\boldsymbol{\beta}$ of the type given by Eq. (39). The tensor $\mathbf{B}$ meets the orthogonality condition $\mathbf{B}:(\boldsymbol{\beta}-\boldsymbol{\alpha})=0$. A representation for $\mathbf{B}$, ensuring that the principal directions of $\boldsymbol{\beta}-\boldsymbol{\alpha}$ remain parallel to those of $\boldsymbol{\sigma}^{\prime}-\boldsymbol{\alpha}$, is

$$
\mathbf{B}=\varphi\left[\underline{\dot{\sigma}}^{\prime}-\frac{\left(\boldsymbol{\sigma}^{\prime}-\boldsymbol{\alpha}\right) \otimes\left(\boldsymbol{\sigma}^{\prime}-\boldsymbol{\alpha}\right)}{\left(\boldsymbol{\sigma}^{\prime}-\boldsymbol{\alpha}\right):\left(\boldsymbol{\sigma}^{\prime}-\boldsymbol{\alpha}\right)}: \underline{\dot{\sigma}}\right],
$$

where 


$$
\varphi=\left[\frac{(\boldsymbol{\beta}-\boldsymbol{\alpha}):(\boldsymbol{\beta}-\boldsymbol{\alpha})}{\left(\boldsymbol{\sigma}^{\prime}-\boldsymbol{\alpha}\right):\left(\boldsymbol{\sigma}^{\prime}-\boldsymbol{\alpha}\right)}\right]^{1 / 2}
$$

Thus, the evolution equation for the rest stress is

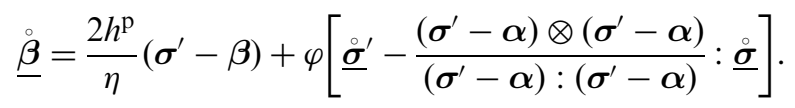

The evolution equation for the back stress $\boldsymbol{\alpha}$, accompanying (48), is in the case of linear kinematic hardening,

$$
\stackrel{\circ}{\boldsymbol{\alpha}}=2 h^{\mathrm{p}} \mathbf{D}^{\mathrm{p}}=\frac{2 h^{\mathrm{p}}}{\eta}\left(\boldsymbol{\sigma}^{\prime}-\boldsymbol{\beta}\right) .
$$

If combined isotropic-kinematic hardening is used, with the yield condition

$$
f=\frac{1}{2}(\boldsymbol{\beta}-\boldsymbol{\alpha}):(\boldsymbol{\beta}-\boldsymbol{\alpha})-k_{\alpha}^{2}(\vartheta)=0,
$$

and with an evolution equation for $\boldsymbol{\alpha}$ corresponding to nonlinear kinematic hardening defined by Eq. (29), the parameter $h^{\mathrm{p}}$ in Eq. (48) is replaced with

$$
h_{*}^{\mathrm{p}}=h_{\alpha}^{\mathrm{p}}+h(1+m) .
$$

Here, $h_{\boldsymbol{\alpha}}^{\mathrm{p}}=\mathrm{d} k_{\boldsymbol{\alpha}} / \mathrm{d} \vartheta$ and $m$ is defined by Eq. (31), in which $\boldsymbol{\sigma}^{\prime}$ is replaced with $\boldsymbol{\beta}$, i.e.

$$
m=\frac{\tau}{2 h} \frac{(\boldsymbol{\beta}-\boldsymbol{\alpha}): \boldsymbol{\alpha}}{[(\boldsymbol{\beta}-\boldsymbol{\alpha}):(\boldsymbol{\beta}-\boldsymbol{\alpha})]^{1 / 2}}=\frac{c}{2 h} \frac{(\boldsymbol{\beta}-\boldsymbol{\alpha}): \boldsymbol{\alpha}}{\sqrt{2} k_{\boldsymbol{\alpha}}} .
$$

The parameters $h$ and $c$ appear in the evolution equation for the back stress $\boldsymbol{\alpha}$, given by Eq. (29).

\subsection{Explicit equation for the rest stress}

Instead of constructing an evolution equation for the objective rate of the rest stress, we may directly construct an explicit equation for the rest stress itself. For example, suppose that principal directions of $\boldsymbol{\beta}$ and $\sigma^{\prime}$ coincide, so that

$$
\boldsymbol{\beta}=\varphi \boldsymbol{\sigma}^{\prime},
$$

where $\varphi$ is an appropriate scalar function. The corresponding plastic rate of deformation is from Eq. (36) 


$$
\mathbf{D}^{\mathrm{p}}=\frac{1}{\eta}(1-\varphi) \boldsymbol{\sigma}^{\prime}
$$

The plastic loading condition $\boldsymbol{\beta}$ : $\left(\boldsymbol{\sigma}^{\prime}-\boldsymbol{\beta}\right)>0$ gives $\varphi(1-\varphi)>0$, i.e.

$$
0<\varphi<1
$$

If the scalar parameter $\varphi$ is defined by

$$
\varphi=\frac{k(\vartheta)}{J_{2}^{1 / 2}}, \quad J_{2}=\frac{1}{2} \sigma^{\prime}: \boldsymbol{\sigma}^{\prime},
$$

where $k=k(\vartheta)$ is the static yield stress in a simple shear test, Eq. (54) reproduces the well-known expression of the Sokolovskii-Malvern-Perzyna viscoplastic overstress theory

$$
\mathbf{D}^{\mathrm{p}}=\frac{1}{\eta}\left(1-\frac{k}{J_{2}^{1 / 2}}\right) \boldsymbol{\sigma}^{\prime}, \quad 1-\frac{k}{J_{2}^{1 / 2}}>0 .
$$

To make the contact with results presented in Section 3.1, we differentiate (53) to obtain

$$
\stackrel{\circ}{\boldsymbol{\beta}}=\dot{\varphi} \boldsymbol{\sigma}^{\prime}+\varphi \underline{\circ}^{\prime}
$$

Since

$$
\varphi^{2}=\frac{\boldsymbol{\beta}: \boldsymbol{\beta}}{\boldsymbol{\sigma}^{\prime}: \boldsymbol{\sigma}^{\prime}}
$$

there follows

$$
\frac{\dot{\varphi}}{\varphi}=\frac{\boldsymbol{\beta}: \underline{\boldsymbol{\beta}}}{\boldsymbol{\beta}: \boldsymbol{\beta}}-\frac{\boldsymbol{\sigma}^{\prime}: \underline{\underline{\boldsymbol{\sigma}}}}{\boldsymbol{\sigma}^{\prime}: \boldsymbol{\sigma}^{\prime}} .
$$

Substituting Eq. (60) into (58) gives

$$
\stackrel{\circ}{\boldsymbol{\beta}}=\frac{\boldsymbol{\beta} \otimes \boldsymbol{\beta}}{\boldsymbol{\beta}: \boldsymbol{\beta}}: \stackrel{\circ}{\boldsymbol{\beta}}+\varphi\left(\underline{\circ}^{\prime}-\frac{\boldsymbol{\sigma}^{\prime} \otimes \boldsymbol{\sigma}^{\prime}}{\boldsymbol{\sigma}^{\prime}: \boldsymbol{\sigma}^{\prime}}: \stackrel{\circ}{\boldsymbol{\sigma}}\right),
$$

which is, in view of Eq. (37), exactly the evolution equation (42).

Suppose next that the static yield surface expands and translates according to combined isotropic-kinematic hardening model considered in Section 2.3, so that Eq. (50) holds. If the loading surface encloses the static yield surface, being also centered at the back stress $\boldsymbol{\alpha}$, then 


$$
\hat{f}=\frac{1}{2}\left(\boldsymbol{\sigma}^{\prime}-\boldsymbol{\alpha}\right):\left(\boldsymbol{\sigma}^{\prime}-\boldsymbol{\alpha}\right)-\hat{k}^{2}=0 .
$$

The current radius of the static yield surface is $k_{\alpha}(\vartheta)$ and the current radius of the loading surface is $\hat{k}$. In this model, the equation for the rest stress $\boldsymbol{\beta}$ is

$$
\boldsymbol{\beta}=\varphi \boldsymbol{\sigma}^{\prime}+(1+\varphi) \boldsymbol{\alpha}
$$

This can be equivalently expressed as either of

$$
\boldsymbol{\sigma}^{\prime}-\boldsymbol{\beta}=(1-\varphi)\left(\boldsymbol{\sigma}^{\prime}-\boldsymbol{\alpha}\right), \quad \boldsymbol{\sigma}^{\prime}-\boldsymbol{\alpha}=\frac{1}{\varphi}(\boldsymbol{\beta}-\boldsymbol{\alpha})
$$

When Eq. (63) is substituted into Eq. (36), the plastic rate of deformation becomes

$$
\mathbf{D}^{\mathrm{p}}=\frac{1}{\eta}(1-\varphi)\left(\boldsymbol{\sigma}^{\prime}-\boldsymbol{\alpha}\right)
$$

The loading condition,

$$
(\boldsymbol{\beta}-\boldsymbol{\alpha}):\left(\boldsymbol{\sigma}^{\prime}-\boldsymbol{\beta}\right)>0,
$$

becomes

$$
\varphi(1-\varphi)\left(\boldsymbol{\sigma}^{\prime}-\boldsymbol{\alpha}\right):\left(\boldsymbol{\sigma}^{\prime}-\boldsymbol{\alpha}\right)>0
$$

This is clearly satisfied when $\varphi$ is in the range $0<\varphi<1$. By defining $\varphi$ to be

$$
\varphi=\frac{k_{\boldsymbol{\alpha}}}{\hat{k}}=[(\boldsymbol{\beta}-\boldsymbol{\alpha}):(\boldsymbol{\beta}-\boldsymbol{\alpha})]^{1 / 2} /\left[\left(\boldsymbol{\sigma}^{\prime}-\boldsymbol{\alpha}\right):\left(\boldsymbol{\sigma}^{\prime}-\boldsymbol{\alpha}\right)\right]^{1 / 2},
$$

the plastic rate of deformation (65) can be written as

$$
\mathbf{D}^{\mathrm{p}}=\frac{1}{\eta}\left(1-\frac{k_{\alpha}}{\hat{k}}\right)\left(\boldsymbol{\sigma}^{\prime}-\boldsymbol{\alpha}\right), \quad 1-\frac{k_{\alpha}}{\hat{k}}>0 .
$$

The total rate of deformation is obtained by summing the elastic and plastic rates of deformation,

$$
\mathbf{D}=\left(\frac{1}{2 \mu} \boldsymbol{J}+\frac{1}{3 \kappa} \boldsymbol{K}\right): \underline{\circ}+\frac{1}{\eta}\left(1-\frac{k_{\boldsymbol{\alpha}}}{\hat{k}}\right)\left(\boldsymbol{\sigma}^{\prime}-\boldsymbol{\alpha}\right) .
$$

The inverted form is 


$$
\underline{\circ}=(2 \mu \boldsymbol{J}+3 \kappa \boldsymbol{K}): \mathbf{D}-\frac{2 \mu}{\eta}\left(1-\frac{k_{\boldsymbol{\alpha}}}{\hat{k}}\right)\left(\boldsymbol{\sigma}^{\prime}-\boldsymbol{\alpha}\right) .
$$

An accompanying evolution equation for the back stress $\boldsymbol{\alpha}$, of nonlinear kinematic hardening type, is given by Eq. (29).

Returning to Eq. (69), the plastic rate of deformation can be rewritten as

$$
\mathbf{D}^{\mathrm{p}}=\frac{k_{\boldsymbol{\alpha}}}{\eta} \frac{\hat{k}-k_{\boldsymbol{\alpha}}}{k_{\boldsymbol{\alpha}}} \frac{\boldsymbol{\sigma}^{\prime}-\boldsymbol{\alpha}}{\hat{k}}, \quad \hat{k}>k_{\boldsymbol{\alpha}} .
$$

This is recast in this form to make the generalization which incorporates a drag strength $d$, such that

$$
\mathbf{D}^{\mathrm{p}}=g(s) \frac{\boldsymbol{\sigma}^{\prime}-\boldsymbol{\alpha}}{\left\|\boldsymbol{\sigma}^{\prime}-\boldsymbol{\alpha}\right\|}, \quad s=\frac{\left\langle\left\|\boldsymbol{\sigma}^{\prime}-\boldsymbol{\alpha}\right\|-\sqrt{2} k_{\boldsymbol{\alpha}}\right\rangle}{d},
$$

where

$$
\left\|\sigma^{\prime}-\alpha\right\|=\left[\left(\sigma^{\prime}-\alpha\right):\left(\sigma^{\prime}-\alpha\right)\right]^{1 / 2}=\sqrt{2} \hat{k}
$$

The Macauley brackets are denoted by \langle\rangle , with $\langle\psi\rangle=0$ if $\psi<0$ and $\langle\psi\rangle=\psi$ if $\psi \geqslant 0$. The scalar function $g$ typically depends nonlinearly on $s$ and has a dimension of the strain rate $\left(t^{-1}\right)$. Evidently, $\left\|\mathbf{D}^{\mathrm{p}}\right\|=g(s)$. The constitutive structure (73) has been frequently employed in recent studies of viscoplastic response (e.g. Chaboche, 1989; Bammann, 1990; McDowell, 1992; Freed et al., 1991). The drag strength $d$ can be either constant, or an evolving parameter. In the latter case, its appropriate evolution equation must be additionally supplied. For details, see Chaboche $(1993,1996)$ and Freed and Walker (1993).

\section{Modified viscoplastic model and evolution equation for the rest stress}

The evolution equations for the rest stress (42) or (48) were derived by recognition of the mathematical representation of the tensor $\mathbf{B}$ arising from its orthogonality property with the tensors $\boldsymbol{\beta}$ or $\boldsymbol{\beta}-\boldsymbol{\alpha}$, and from the requirement for continuing coaxiality of the tensors $\boldsymbol{\sigma}^{\prime}$ and $\boldsymbol{\beta}$, or $\boldsymbol{\sigma}^{\prime}-\boldsymbol{\beta}$ and $\boldsymbol{\beta}-\boldsymbol{\alpha}$, respectively. We now present a derivation of another evolution equation for the rest stress, which is not characterized by such coaxiality, and which is based on a three-dimensional extension of the one-dimensional model depicted in Fig. 3. A parallel connection of an elastoplastic and viscous element is attached in series to an elastic element. The elastic part of the strain rate is $\dot{\epsilon}^{\mathrm{e}}=\dot{\sigma} / E$. The inelastic part of the strain rate can be calculated from either of 


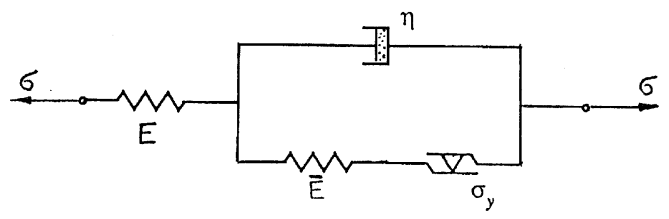

Fig. 3. One-dimensional viscoplastic model with a dashpot of viscosity $\eta$ connected in parallel with an elastoplastic spring-friction element $\left(\bar{E}, \sigma_{Y}\right)$. The elastic modulus of an additional spring element attached in series is $E$.

$$
\dot{\epsilon}^{\mathrm{p}}=\frac{1}{\eta}\left(\sigma-\sigma^{\mathrm{p}}\right)=\left(\frac{1}{\bar{E}}+\frac{1}{H^{\mathrm{p}}}\right) \dot{\sigma}^{\mathrm{p}},
$$

provided that $\sigma^{\mathrm{p}}=\sigma_{Y}$ (and $\left.\dot{\sigma}^{p}>0\right)$. If $\sigma^{\mathrm{p}}<\sigma_{Y}\left(\right.$ or $\sigma^{\mathrm{p}}=\sigma_{Y}$, but $\left.\dot{\sigma}^{\mathrm{p}}<0\right)$, Eq. (75) is replaced with

$$
\dot{\epsilon}^{\mathrm{p}}=\frac{1}{\eta}\left(\sigma-\sigma^{\mathrm{p}}\right)=\frac{1}{\bar{E}} \dot{\sigma}^{\mathrm{p}}
$$

Consequently, the total strain and stress rates are

$$
\begin{aligned}
& \dot{\epsilon}=\frac{1}{E} \dot{\sigma}+\frac{1}{\eta}\left(\sigma-\sigma^{\mathrm{p}}\right), \\
& \dot{\sigma}=E \dot{\epsilon}-\frac{E}{\eta}\left(\sigma-\sigma^{\mathrm{p}}\right) .
\end{aligned}
$$

The $\sigma^{\mathrm{p}}$ part of the stress is determined by integration from

$$
\dot{\sigma}^{\mathrm{p}}=\frac{1}{\eta}\left(\bar{E}-\frac{\bar{E}}{1+H^{\mathrm{p}} / \bar{E}}\right)\left(\sigma-\sigma^{\mathrm{p}}\right), \quad \sigma^{\mathrm{p}}=\sigma_{Y} .
$$

If $\sigma^{\mathrm{p}}<\sigma_{\mathrm{Y}}$, then

$$
\dot{\sigma}^{\mathrm{p}}=\frac{\bar{E}}{\eta}\left(\sigma-\sigma^{\mathrm{p}}\right)
$$

A three-dimensional generalization of Eq. (77) is

$$
\mathbf{D}=\left(\frac{1}{2 \mu} \boldsymbol{J}+\frac{1}{3 \kappa} \boldsymbol{K}\right): \stackrel{\circ}{\boldsymbol{\sigma}}+\frac{1}{\eta}\left(\boldsymbol{\sigma}^{\prime}-\boldsymbol{\beta}\right),
$$

where $\boldsymbol{\beta}$ is again the rest stress. Similarly, Eq. (78) becomes 


$$
\underline{\circ}=(2 \mu \boldsymbol{J}+3 \kappa \boldsymbol{K}): \mathbf{D}-\frac{2 \mu}{\eta}\left(\boldsymbol{\sigma}^{\prime}-\boldsymbol{\beta}\right) .
$$

The generalization of Eq. (75) is

$$
\frac{1}{\eta}\left(\boldsymbol{\sigma}^{\prime}-\boldsymbol{\beta}\right)=\frac{1}{2 \bar{\mu}} \underline{\stackrel{\boldsymbol{\beta}}{ }}+\frac{1}{2 h^{\mathrm{p}}} \frac{\boldsymbol{\beta} \otimes \boldsymbol{\beta}}{\boldsymbol{\beta}: \boldsymbol{\beta}}: \stackrel{\circ}{\boldsymbol{\beta}}
$$

which preserves the deviatoric character of the tensors on both sides of the equation. Solving for the Jaumann rate of the rest stress gives the evolution equation

$$
\stackrel{\circ}{\boldsymbol{\beta}}=\frac{2 \bar{\mu}}{\eta}\left[\boldsymbol{J}-\frac{1}{1+h^{\mathrm{p}} / \bar{\mu}} \frac{\boldsymbol{\beta} \otimes \boldsymbol{\beta}}{\boldsymbol{\beta}: \boldsymbol{\beta}}\right]:\left(\boldsymbol{\sigma}^{\prime}-\boldsymbol{\beta}\right) .
$$

The principal directions of $\boldsymbol{\sigma}^{\prime}$ and $\boldsymbol{\beta}$ in general do not coincide, since $\stackrel{\circ}{\boldsymbol{\beta}}$ is not necessarily codirectional with $\boldsymbol{\beta}$. Indeed, if $\boldsymbol{\sigma}^{\prime}$ would be codirectional with $\boldsymbol{\beta}$, then Eq. (84) would imply that $\boldsymbol{\beta}$ is codirectional with $\boldsymbol{\beta}$, as well. This is in general not the case, because there is an additional contribution to $\underline{\boldsymbol{\beta}}$, perpendicular to $\boldsymbol{\beta}$; see Eq. (61).

Eqs. (83) and (84) hold provided that

$$
\frac{1}{2} \boldsymbol{\beta}: \boldsymbol{\beta}=k^{2}(\vartheta), \quad \stackrel{\circ}{\boldsymbol{\beta}}: \boldsymbol{\beta}>0 .
$$

Otherwise, Eq. (84) is replaced with

$$
\stackrel{\circ}{\boldsymbol{\beta}}=\frac{2 \bar{\mu}}{\eta}\left(\boldsymbol{\sigma}^{\prime}-\boldsymbol{\beta}\right) .
$$

In Eqs. (83)-(85) the modulus $h^{\mathrm{p}}$ is defined by $h^{p}=\mathrm{d} k / \mathrm{d} \vartheta$, where

$$
\vartheta=\int{ }_{0}^{t}\left(2 \boldsymbol{D}^{\mathrm{p}}: \boldsymbol{D}^{\mathrm{p}}\right)^{1 / 2} \mathrm{~d} t
$$

The plastic rate of deformation, associated with elastoplastic material segments corresponding to $\left(\bar{E}, \sigma_{\mathrm{Y}}\right)$ element in Fig. 3, is given by

$$
\boldsymbol{D}^{\mathrm{p}}=\frac{1}{\eta}\left(\boldsymbol{\sigma}^{\prime}-\boldsymbol{\beta}\right)-\frac{1}{2 \bar{\mu}} \stackrel{\circ}{\boldsymbol{\beta}}=\frac{1}{\eta} \frac{1}{1+h^{\mathrm{p}} / \bar{\mu}} \frac{\boldsymbol{\beta} \otimes \boldsymbol{\beta}}{\boldsymbol{\beta}: \boldsymbol{\beta}}:\left(\boldsymbol{\sigma}^{\prime}-\boldsymbol{\beta}\right) .
$$

In view of (84) it follows that $\boldsymbol{D}^{\mathrm{p}}$, but not $\boldsymbol{D}^{\mathrm{p}}=\left(\boldsymbol{\sigma}^{\prime}-\boldsymbol{\beta}\right) / \eta$ is codirectional with $\boldsymbol{\beta}$. It can also be easily verified that the evolution equation (84) satisfies the consistency condition 


$$
\boldsymbol{\beta}: \stackrel{\circ}{\boldsymbol{\beta}}=2 k h^{\mathrm{p}}\left(2 \boldsymbol{D}^{\mathrm{p}}: \boldsymbol{D}^{\mathrm{p}}\right)^{1 / 2},
$$

which follows from Eq. (85).

In the case of linear kinematic hardening, the evolution equation for $\boldsymbol{\beta}$, replacing Eq. (84), is

$$
\stackrel{\circ}{\boldsymbol{\beta}}=\frac{2 \bar{\mu}}{\eta}\left[\boldsymbol{J}-\frac{1}{1+h^{\mathrm{p}} / \bar{\mu}} \frac{(\boldsymbol{\beta}-\boldsymbol{\alpha}) \otimes(\boldsymbol{\beta}-\boldsymbol{\alpha})}{(\boldsymbol{\beta}-\boldsymbol{\alpha}):(\boldsymbol{\beta}-\boldsymbol{\alpha})}\right]:\left(\boldsymbol{\sigma}^{\prime}-\boldsymbol{\beta}\right) .
$$

The accompanying evolution equation for the back stress $\boldsymbol{\alpha}$ is

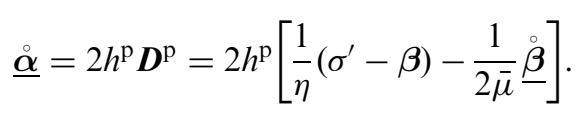

Upon substitution of (90), this can be written as

$$
\underline{\stackrel{\circ}{\alpha}}=\frac{1}{\eta} \frac{2 h^{\mathrm{p}}}{1+h^{\mathrm{p}} / \bar{\mu}} \frac{(\boldsymbol{\beta}-\boldsymbol{\alpha}) \otimes(\boldsymbol{\beta}-\boldsymbol{\alpha})}{(\boldsymbol{\beta}-\boldsymbol{\alpha}):(\boldsymbol{\beta}-\boldsymbol{\alpha})}:\left(\boldsymbol{\sigma}^{\prime}-\boldsymbol{\beta}\right) .
$$

If combined isotropic-kinematic hardening is used, with the yield condition (50) and the evolution equation for $\boldsymbol{\alpha}$ given by (29), the parameter $h^{\mathrm{p}}$ in Eq. (90) should be replaced with the parameter $h_{*}^{\text {p }}$ of Eq. (51), while Eq. (92) generalizes to

$$
\underline{\stackrel{\alpha}{\alpha}}=\frac{1}{\eta} \frac{2 h}{1+h_{*}^{\mathrm{p}} / \bar{\mu}}\left(\boldsymbol{\beta}-\boldsymbol{\alpha}+\frac{c}{\sqrt{2}} \frac{k_{\alpha}}{h} \boldsymbol{\alpha}\right) \frac{(\boldsymbol{\beta}-\boldsymbol{\alpha}):\left(\boldsymbol{\sigma}^{\prime}-\boldsymbol{\beta}\right)}{(\boldsymbol{\beta}-\boldsymbol{\sigma}):(\boldsymbol{\beta}-\boldsymbol{\alpha})} .
$$

An equivalent representation, explicitly involving the parameter $m$ from Eq. (52), is

$$
\underline{\stackrel{\circ}{\alpha}}=\frac{1}{\eta} \frac{2 h}{1+h_{*}^{\mathrm{p}} / \bar{\mu}}\left[\frac{(\boldsymbol{\beta}-\boldsymbol{\alpha}) \otimes(\boldsymbol{\beta}-\boldsymbol{\alpha})}{(\boldsymbol{\beta}-\boldsymbol{\alpha}):(\boldsymbol{\beta}-\boldsymbol{\alpha})}+m \frac{\boldsymbol{\alpha} \otimes(\boldsymbol{\beta}-\boldsymbol{\alpha})}{\boldsymbol{\alpha}:(\boldsymbol{\beta}-\boldsymbol{\alpha})}\right]:\left(\boldsymbol{\sigma}^{\prime}-\boldsymbol{\beta}\right) .
$$

\section{Evolution of the rest stress in the Bordenhagen-Stout-Gray viscoplastic model}

Bardenhagen et al. (1997) suggested a viscoplastic model that is a three-dimensional generalization of the one-dimensional model shown in Fig. 4. The viscoelastic Maxwell element $(\tilde{E}, \eta)$ is connected in parallel to an elastoplastic element $\left(\bar{E}, \sigma_{Y}\right)$. The stress is carried by both elements, $\sigma=\sigma^{\mathrm{v}}+\sigma^{\mathrm{p}}$. The stress rate can be written as the sum of viscous and plastic parts, such that

$$
\dot{\sigma}=\dot{\sigma}^{\mathrm{v}}+\dot{\sigma}^{\mathrm{p}}
$$




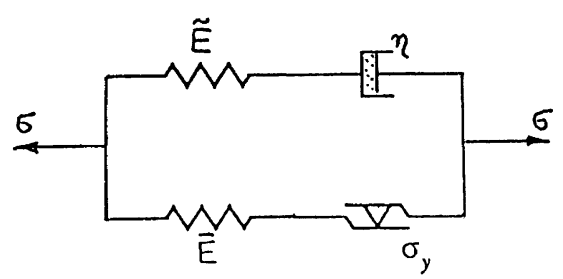

Fig. 4. One-dimensional viscoplastic model in which a viscoelastic spring-dashpot element $(\tilde{E}, \eta)$ is connected in parallel to an elastoplastic spring-friction element $\left(\bar{E}, \sigma_{Y}\right)$.

The two elements deform equally, and the rate of strain can be expressed as either

$$
\dot{\epsilon}=\frac{1}{\tilde{E}} \dot{\sigma}^{\mathrm{v}}+\frac{1}{\eta} \sigma^{\mathrm{v}}
$$

or

$$
\dot{\epsilon}=\left(\frac{1}{\bar{E}}+\frac{1}{H^{\mathrm{p}}}\right) \dot{\sigma}^{\mathrm{p}}, \quad \text { for } \quad \sigma^{\mathrm{p}}=\sigma_{Y} \quad \text { and } \quad \dot{\sigma}^{\mathrm{p}}>0
$$

For $\sigma^{\mathrm{p}}<\sigma_{\mathrm{Y}}$, or $\sigma^{\mathrm{p}}=\sigma_{\mathrm{Y}}$ and $\sigma^{\mathrm{p}} \leqslant 0$, no plastic deformation takes place and, instead of (97), we have $\dot{\epsilon}=\dot{\sigma}^{\mathrm{p}} / \bar{E}$. The viscous part of the stress rate is, from Eq. (96),

$$
\dot{\sigma}^{\mathrm{v}}=\tilde{E} \dot{\epsilon}-\frac{\tilde{E}}{\eta} \sigma^{\mathrm{v}}=\tilde{E} \dot{\epsilon}-\frac{\tilde{E}}{\eta}\left(\sigma-\sigma^{\mathrm{p}}\right) .
$$

The plastic part is, from Eq. (97),

$$
\dot{\sigma}^{\mathrm{p}}=\left(\frac{1}{\bar{E}}+\frac{1}{H^{\mathrm{p}}}\right)^{-1} \dot{\epsilon}=\left(\bar{E}-\frac{\bar{E}}{1+H^{\mathrm{p}} / \bar{E}}\right) \dot{\epsilon}
$$

Substitution of Eqs. (98) and (99) into Eq. (95) gives

$$
\dot{\sigma}=\left(E-\frac{\bar{E}}{1+H^{\mathrm{p}} / \bar{E}}\right) \dot{\epsilon}-\frac{\tilde{E}}{\eta}\left(\sigma-\sigma^{\mathrm{p}}\right)
$$

The modulus $E=\tilde{E}+\bar{E}$ is the overall, equivalent elastic modulus of the material. The plastic part of stress $\sigma^{\mathrm{p}}$ is determined by integration from Eq. (99) with the initial condition $\sigma^{\mathrm{p}}=0$ when $\epsilon=0$. Only elastic deformation takes place in the elastoplastic element for $\sigma^{\mathrm{p}}<\sigma_{Y}$. 
A three-dimensional generalization of the stress rate decomposition in Eq. (95) is

$$
\underline{\circ}=\underline{\circ}^{\mathrm{v}}+\underline{\circ}^{\mathrm{p}}
$$

The viscous part of the rate of stress is, by extending Eq. (98),

$$
\underline{\sigma}^{v}=(2 \tilde{\mu} \boldsymbol{J}+3 \tilde{\kappa} \boldsymbol{K}): \mathbf{D}-\frac{2 \tilde{\mu}}{\eta}\left(\boldsymbol{\sigma}^{\prime}-\boldsymbol{\beta}\right)
$$

The deviatoric part of the plastic stress $\boldsymbol{\sigma}^{\mathrm{p}}$ is denoted by $\boldsymbol{\beta}$. Since $\underline{\boldsymbol{\sigma}}^{\mathrm{v}}$ in Eq. (102) depends on the stress difference $\left(\boldsymbol{\sigma}^{\prime}-\boldsymbol{\beta}\right)$, we shall again refer to $\boldsymbol{\beta}$ as the rest stress. The plastic part of the rate of stress is obtained by generalizing Eq. (99) as

$$
\stackrel{\circ}{\mathrm{o}}^{\mathrm{p}}=\left(2 \bar{\mu} \boldsymbol{J}+3 \bar{\kappa} \boldsymbol{K}-\frac{2 \bar{\mu}}{1+h^{\mathrm{p}} / \bar{\mu}} \frac{\boldsymbol{\beta} \otimes \boldsymbol{\beta}}{\boldsymbol{\beta}: \boldsymbol{\beta}}\right): \mathbf{D}, \quad \boldsymbol{\beta}: \mathbf{D}>0 .
$$

The rest stress is assumed to be on the yield surface

$$
\frac{1}{2} \boldsymbol{\beta} \quad \boldsymbol{\beta}=k^{2}(\boldsymbol{\vartheta})
$$

Here,

$$
\dot{v}=\left(2 \boldsymbol{D}^{\mathrm{p}}: \boldsymbol{D}^{\mathrm{p}}\right)^{1 / 2}=\frac{\sqrt{2}}{1+h^{\mathrm{p}} / \bar{\mu}} \frac{\boldsymbol{\beta}: \mathbf{D}}{(\boldsymbol{\beta}: \boldsymbol{\beta})^{1 / 2}},
$$

where $D^{\mathrm{p}}$ is the plastic part of the rate of deformation due to stress rate $\underline{o}^{\mathrm{p}}$. This is

$$
D^{\mathrm{p}}=\frac{1}{2 h^{\mathrm{p}}} \frac{\boldsymbol{\beta} \otimes \boldsymbol{\beta}}{\boldsymbol{\beta}: \boldsymbol{\beta}}: \stackrel{\circ}{\mathrm{p}}^{\mathrm{p}}=\frac{1}{1+h^{\mathrm{p}} / \bar{\mu}} \frac{\boldsymbol{\beta} \otimes \boldsymbol{\beta}}{\boldsymbol{\beta}: \boldsymbol{\beta}}: \mathbf{D} .
$$

If (104) holds, but $\boldsymbol{\beta}: \mathbf{D} \leqslant 0$, or if $\boldsymbol{\beta}: \boldsymbol{\beta}<2 \mathrm{k}^{2}(\vartheta)$, Eq. (103) is replaced with

$$
\underline{\circ}^{\mathrm{p}}=(2 \bar{\mu} \boldsymbol{J}+3 \bar{\kappa} \boldsymbol{K}): \mathbf{D} .
$$

The evolution equation for the rest stress follows from Eq. (103), by taking its deviatoric part,

$$
\stackrel{\circ}{\boldsymbol{\beta}}=2 \bar{\mu}\left(\boldsymbol{J}-\frac{1}{1+h^{\mathrm{p}} / \bar{\mu}} \frac{\boldsymbol{\beta} \otimes \boldsymbol{\beta}}{\boldsymbol{\beta}: \boldsymbol{\beta}}\right): \mathbf{D}, \quad \boldsymbol{\beta}: \mathbf{D}>0 .
$$

If (104) holds, but $\boldsymbol{\beta}: \mathbf{D} \leqslant 0$, or if $\boldsymbol{\beta}: \boldsymbol{\beta}<2 \mathrm{k}^{2}(\vartheta)$, Eq. (108) is replaced with

$$
\stackrel{\stackrel{\beta}{\boldsymbol{\beta}}}{=}=2 \bar{\mu} \mathbf{D} .
$$


The overall constitutive structure follows by substituting Eqs. (102) and (103) into Eq. (101),

$$
\underline{\stackrel{\sigma}{\sigma}}=\left(\boldsymbol{L}^{e}-\frac{2 \mu}{1+h^{\mathrm{p}} / \bar{\mu}} \frac{\boldsymbol{\beta} \otimes \boldsymbol{\beta}}{\boldsymbol{\beta}: \boldsymbol{\beta}}\right): \mathbf{D}-2 \frac{\tilde{\mu}}{\eta}\left(\boldsymbol{\sigma}^{\prime}-\boldsymbol{\beta}\right), \quad \boldsymbol{\beta}: \mathbf{D}>0 .
$$

Otherwise,

$$
\underline{\stackrel{\circ}{\boldsymbol{\sigma}}}=\boldsymbol{L}^{e}: \mathbf{D}-\frac{2 \tilde{\mu}}{\eta}\left(\boldsymbol{\sigma}^{\prime}-\boldsymbol{\beta}\right)
$$

The overall elastic stiffness tensor is

$$
\boldsymbol{L}^{e}=2 \mu \boldsymbol{J}+3 \kappa \boldsymbol{K}
$$

where

$$
\mu=\tilde{\mu}+\bar{\mu} \quad \kappa=\tilde{\kappa}+\bar{\kappa}
$$

These are the true elastic shear and bulk moduli of the material. The modulus $\bar{\mu}<\mu$ in Eq. (110) may be viewed as an additional material parameter, while $\tilde{\mu}=\mu-\bar{\mu}$.

The considered model can be extended by replacing the isotropic hardening yield condition for the rest stress in Eq. (104) with the kinematic hardening condition

$$
\frac{1}{2}(\boldsymbol{\beta}-\boldsymbol{\alpha}):(\boldsymbol{\beta}-\boldsymbol{\alpha})=k_{0}^{2}, \quad k_{0}=\text { const. }
$$

where $\boldsymbol{\alpha}$ is the back stress. The plastic part of the rate of stress is in this case

$$
\underline{\underline{\sigma}}^{\mathrm{p}}=\left[2 \bar{\mu} \boldsymbol{J}+3 \bar{\kappa} \boldsymbol{K}-\frac{2 \bar{\mu}}{1+h^{\mathrm{p}} / \bar{\mu}} \frac{(\boldsymbol{\beta}-\boldsymbol{\alpha}) \otimes(\boldsymbol{\beta}-\boldsymbol{\alpha})}{(\boldsymbol{\beta}-\boldsymbol{\alpha}):(\boldsymbol{\beta}-\boldsymbol{\alpha})}\right]: \mathbf{D},
$$

with $(\boldsymbol{\beta}-\boldsymbol{\alpha}): \mathbf{D}>0$. The corresponding evolution equation for $\boldsymbol{\beta}$ is

$$
\stackrel{\stackrel{\boldsymbol{\beta}}{ }}{=} 2 \bar{\mu}\left[\boldsymbol{J}-\frac{1}{1+h^{\mathrm{p}} / \bar{\mu}} \frac{(\boldsymbol{\beta}-\boldsymbol{\alpha}) \otimes(\boldsymbol{\beta}-\boldsymbol{\alpha})}{(\boldsymbol{\beta}-\boldsymbol{\alpha}):(\boldsymbol{\beta}-\boldsymbol{\alpha})}\right]: \quad \mathbf{D}, \quad(\boldsymbol{\beta}-\boldsymbol{\alpha}): \mathbf{D}>0 .
$$

Otherwise, Eqs. (107) and (109) apply. The overall constitutive structure is consequently

$$
\underline{\dot{\sigma}}=\left[\boldsymbol{L}-\frac{2 \bar{\mu}}{1+h^{\mathrm{p}} / \bar{\mu}} \frac{(\boldsymbol{\beta}-\boldsymbol{\alpha}) \otimes(\boldsymbol{\beta}-\boldsymbol{\alpha})}{(\boldsymbol{\beta}-\boldsymbol{\alpha}):(\boldsymbol{\beta}-\boldsymbol{\alpha})}\right] \quad \mathbf{D}-\frac{2 \bar{\mu}}{\eta},\left(\sigma^{\prime}-\boldsymbol{\beta}\right),
$$

with $(\boldsymbol{\beta}-\boldsymbol{\alpha}): \mathbf{D}>0$. 
Otherwise, Eq. (111) applies. For linear kinematic hardening the evolution of the back stress is

$$
\underline{\stackrel{\alpha}{\alpha}}=2 h^{\mathrm{p}} \boldsymbol{D}^{\mathrm{p}}
$$

where $\boldsymbol{D}^{\mathrm{p}}$ is plastic part of the rate of deformation due to stress rate $\underline{\boldsymbol{\sigma}}^{\mathrm{p}}$. This is

$$
D^{\mathrm{p}}=\frac{1}{2 h^{\mathrm{p}}} \frac{(\boldsymbol{\beta}-\boldsymbol{\alpha}) \otimes(\boldsymbol{\beta}-\boldsymbol{\alpha})}{(\boldsymbol{\beta}-\boldsymbol{\alpha}):(\boldsymbol{\beta}-\boldsymbol{\alpha})}: \underline{\circ}^{\mathrm{p}}=\frac{1}{1+h^{\mathrm{p}} / \bar{\mu}} \frac{(\boldsymbol{\beta}-\boldsymbol{\alpha}) \otimes(\boldsymbol{\beta}-\boldsymbol{\alpha})}{(\boldsymbol{\beta}-\boldsymbol{\alpha}):(\boldsymbol{\beta}-\boldsymbol{\alpha})}: \mathbf{D},
$$

by extending the results from Eq. (19). The evolution equation for the back stress (118) is accordingly

$$
\stackrel{\circ}{\boldsymbol{\alpha}}=\frac{2 h^{\mathrm{p}}}{1+h^{\mathrm{p}} / \bar{\mu}} \frac{(\boldsymbol{\beta}-\boldsymbol{\alpha}) \otimes(\boldsymbol{\beta}-\boldsymbol{\alpha})}{(\boldsymbol{\beta}-\boldsymbol{\alpha}):(\boldsymbol{\beta}-\boldsymbol{\alpha})}: \mathbf{D}, \quad(\boldsymbol{\beta}-\boldsymbol{\alpha}): \mathbf{D}>0 .
$$

This representation can also be deduced directly from Eq. (28), by appropriate identification of stress tensors and material parameters.

If combined isotropic-kinematic hardening is used, with the yield condition (50) and the evolution equation for $\boldsymbol{\alpha}$ of nonlinear kinematic hardening type

$$
\underline{\stackrel{\alpha}{\alpha}}=2 h \boldsymbol{D}^{\mathrm{p}}+c \boldsymbol{\alpha}\left(\boldsymbol{D}^{\mathrm{p}}: \boldsymbol{D}^{\mathrm{p}}\right)^{1 / 2},
$$

then the parameter $h^{\mathrm{p}}$ in Eqs. (115)-(117) and (119) is replaced with the parameter $\mathrm{h}_{*}^{\mathrm{p}}$ of Eq. (51). Furthermore, Eq. (120) generalizes to

$$
\stackrel{\circ}{\underline{\alpha}}=\frac{2 h}{1+h_{*}^{\mathrm{p}} \bar{\mu}}\left(\boldsymbol{\beta}-\boldsymbol{\alpha}+\frac{C}{\sqrt{2}} \frac{k_{\boldsymbol{\alpha}}}{h} \boldsymbol{\alpha}\right) \frac{(\boldsymbol{\beta}-\boldsymbol{\alpha}): \mathbf{D}}{(\boldsymbol{\beta}-\boldsymbol{\alpha}):(\boldsymbol{\beta}-\boldsymbol{\alpha})} \quad, \quad(\boldsymbol{\beta}-\boldsymbol{\alpha}): \mathbf{D}>0 .
$$

An equivalent representation, involving the parameter $\mathbf{m}$ of Eq. (52), is

$$
\underline{\dot{\alpha}}=\frac{2 h}{1+h_{*}^{\mathrm{p}} \bar{\mu}}\left[\frac{(\boldsymbol{\beta}-\boldsymbol{\alpha}) \otimes(\boldsymbol{\beta}-\boldsymbol{\alpha})}{(\boldsymbol{\beta}-\boldsymbol{\alpha}):(\boldsymbol{\beta}-\boldsymbol{\alpha})}+m \frac{\boldsymbol{\alpha} \otimes(\boldsymbol{\beta}-\boldsymbol{\alpha})}{\boldsymbol{\alpha}:(\boldsymbol{\beta}-\boldsymbol{\alpha})}\right]: \mathbf{D} .
$$

\subsection{Viscoplastic rate of deformation}

With the help of Eqs. (20) and (21), it is straightforward to invert Eq. (110) and obtain

$$
\mathbf{D}=\left(\frac{1}{2 \mu} \boldsymbol{J}+\frac{1}{3 \kappa} \boldsymbol{K}+\frac{\psi}{2 \mu} \frac{\boldsymbol{\beta} \otimes \boldsymbol{\beta}}{\boldsymbol{\beta}: \boldsymbol{\beta}}\right): \frac{\circ}{\boldsymbol{\sigma}}+\frac{1}{\eta} \frac{\tilde{\mu}}{\mu}\left[\sigma^{\prime}-\boldsymbol{\beta}+\psi \frac{\boldsymbol{\beta}:\left(\sigma^{\prime}-\boldsymbol{\beta}\right)}{\boldsymbol{\beta}: \boldsymbol{\beta}}\right] .
$$

The scalar parameter $\psi$ is 


$$
\psi=\frac{\bar{\mu} / \mu}{h^{\mathrm{p}} / \bar{\mu}+\tilde{\mu} / \mu} .
$$

By taking the trace product of (124) with $\boldsymbol{\beta}$, and by imposing the condition $\boldsymbol{\beta}: \mathbf{D}>0$ of Eq. (110), we find that Eq. (124) applies when $\boldsymbol{\beta}$ satisfies (104) and

$$
\boldsymbol{\beta}: \underline{\stackrel{\circ}{\sigma}}>-\frac{2}{\eta} \tilde{\mu}\left[\boldsymbol{\beta}:\left(\sigma^{\prime}-\boldsymbol{\beta}\right)\right]
$$

Otherwise, Eq. (124) is replaced with

$$
\mathbf{D}=\left(\frac{1}{2 \mu} \boldsymbol{J}+\frac{1}{3 \kappa} \boldsymbol{K}\right): \underline{\dot{\sigma}}+\frac{1}{\eta} \frac{\tilde{\mu}}{\mu}\left(\sigma^{\prime}-\boldsymbol{\beta}\right) .
$$

The portion of the total rate of deformation, given by

$$
\mathbf{D}^{e}=\left(\frac{1}{2 \mu} \boldsymbol{J}+\frac{1}{3 \kappa} \boldsymbol{K}\right): \underline{\stackrel{\circ}{ }}
$$

can be interpreted as the elastic rate of deformation that would occur if both viscous and plastic deformation mechanisms were momentarily frozen. The remaining part of the rate of deformation in Eq. (124) is the viscoplastic part,

$$
\mathbf{D}^{\mathrm{p}}=\frac{1}{\eta} \frac{\tilde{\mu}}{\mu}\left(\boldsymbol{\sigma}^{\prime}-\boldsymbol{\beta}\right)+\frac{\psi}{2 \mu} \frac{\boldsymbol{\beta} \otimes \boldsymbol{\beta}}{\boldsymbol{\beta}: \boldsymbol{\beta}}:\left[\underline{\circ}+\frac{2 \tilde{\mu}}{\eta}\left(\sigma^{\prime}-\boldsymbol{\beta}\right)\right] .
$$

Both rate-dependent viscous and rate-independent plastic processes simultaneously take place and contribute to $\mathbf{D}^{\mathrm{p}}$ in Eq. (129). If the current state of stress is such that the rest stress $\boldsymbol{\beta}$ is inside the yield surface (104), or if it is on the surface (104) but

$$
\boldsymbol{\beta}: \underline{\boldsymbol{\sigma}} \leqslant-\frac{2}{\eta} \tilde{\mu}\left[\boldsymbol{\beta}:\left(\boldsymbol{\sigma}^{\prime}-\boldsymbol{\beta}\right)\right]
$$

the inelastic rate of deformation is solely due to the viscous contribution, i.e.

$$
\mathbf{D}^{\mathrm{p}}=\frac{1}{\eta} \frac{\tilde{\mu}}{\mu}\left(\boldsymbol{\sigma}^{\prime}-\boldsymbol{\beta}\right)
$$

In the case of linear kinematic hardening, the elastic part of the rate of deformation is still given by Eq. (125), but the viscoplastic part is governed by

$$
\mathbf{D}^{\mathrm{p}}=\frac{1}{\eta} \frac{\tilde{\mu}}{\mu}\left(\boldsymbol{\sigma}^{\prime}-\boldsymbol{\beta}\right)+\frac{\psi}{2 \mu} \frac{(\boldsymbol{\beta}-\boldsymbol{\alpha}) \otimes(\boldsymbol{\beta}-\boldsymbol{\alpha})}{(\boldsymbol{\beta}-\boldsymbol{\alpha}):(\boldsymbol{\beta}-\boldsymbol{\alpha})}:\left[\underline{\dot{\sigma}}+\frac{2 \tilde{\mu}}{\eta}\left(\sigma^{\prime}-\boldsymbol{\beta}\right)\right] .
$$


For combined isotropic-nonlinear kinematic hardening, the parameter $\psi$ is specified by Eq. (125), in which $h^{\mathrm{p}}$ is replaced with $h_{*}^{\mathrm{p}}$.

\section{Conclusion}

The objective of this paper is to examine a tensorial structure of an evolution equation for the rest stress in viscoplastic modeling of rate-dependent material response. Three different representations of the evolution equation are derived, under certain assumptions, rather than postulated. This was accomplished by suggested threedimensional generalization of several one-dimensional rheological models. In the case of linear kinematic hardening with the back stress $\boldsymbol{\alpha}$, the evolution equations for the rest stress $\boldsymbol{\beta}$ are given by Eqs. (48), (90) and (116). Each of these equations may be suitable to describe the growth of the rest stress, depending on the particular material model and the range of application. With an appropriately specified viscosity function $\eta$, numerical study is needed to compare their predictions in high-strain rate, creep, recovery and relaxation tests. In addition to isotropic and linear kinematic hardening models, nonlinear kinematic hardening and combined isotropic-nonlinear kinematic hardening are included in the analysis. In the latter three cases, an evolution equation for the back stress is incorporated in the constitutive framework, which enables better description of nonproportional cyclic response. Work is in progress to extend the present isothermal formulation to non-isothermal conditions.

\section{Acknowledgements}

Research funding provided by the Los Alamos National Laboratory is kindly acknowledged. We also thank the reviewers for their comments and suggestions.

\section{References}

Armstrong, P.J., Frederick, C.O., 1966. A mathematical representation of the multiaxial Bauschinger effect, G. E. G. B. Report RD/B/N, 731.

Bammann, D.J., 1990. Modeling temperature and strain rate dependent large deformations of metals. Appl. Mech. Rev. 43, S312-S319.

Bardenhagen, S.G., Stout, M.G., Gray, G.T., 1997. Three-dimensional, finite deformation, viscoplastic constitutive models for polymeric materials. Mech. Mater. 25, 235-253.

Bodner, S.R., Partom, Y., 1975. Constitutive equations for elastic-viscoplastic strain-hardening materials. J. Appl. Mech 42, 385-389.

Chaboche, J.L., 1989. Constitutive equations for cyclic plasticity and cyclic viscoplasticity. Int. J. Plasticity $5,247-302$.

Chaboche, J.L., 1993. Cyclic viscoplastic constitutive equations. Part I: a thermodynamically consistent formulation. J. Appl. Mech 60, 813-821.

Chaboche, J.L., 1996. Unified cyclic viscoplastic constitutive equations: development, capabilities, and thermodynamic framework. In: Krausz, A.S., Krausz, K. (Eds.), Unified Constitutive Laws for Plastic Deformation. Academic Press, San Diego, pp. 1-68. 
Freed, A.D., Chaboche, J.L., Walker, K.P., 1991. A viscoplastic theory with thermodynamic considerations. Acta Mech. 90, 155-174.

Freed, A.D., Walker, K.P., 1993. Viscoplasticity with creep and plasticity bounds. Int. J. Plasticity 9, 213-242.

Hart, E.W., 1970. A phenomenological theory for plastic deformation of polycrystalline metals. Acta Metall. 18, 599-610.

Hill, R., 1965. Continuum micro-mechanics of elastoplastic polycrystals. J. Mech. Phys. Solids 13, 89101.

Johnson, G.R., Cook, W.H., 1983. A constitutive model and data for metals subjected to large strains, high strain rates, and high temperatures. In: Proceedings of the 7th International Symposium on Ballistics, The Hague, The Netherlands, pp. 1-7.

Johnston, W.G., Gilman, J.J., 1959. Dislocation velocities, dislocation densities, and plastic flow in LiF crystals. J. Appl. Phys. 30, 129-144.

Krempl, E., 1996. A small-strain viscoplasticity theory based on overstress. In: Krausz, A.S., Krausz, K. (Eds.), Unified Constitutive Laws for Plastic Deformation. Academic Press, San Diego, pp. 281-318.

Krempl, E., Gleason, J.M., 1996. Isotropic viscoplasticity theory based on overstress (VBO): the influence of the direction of the dynamic recovery term in the growth law of the equilibrium stress. Int. J. Plasticity 12, 719-735.

Krieg, R.D., 1977. Numerical integration of some new unified plasticity-creep formulations. In: Proc. 4th SMIRT Conference, San Francisco, Paper M6/4.

Lee, E.H., Mallett, R.L., Wertheimer, T.B., 1983. Stress analysis for anisotropic hardening in finitedeformation plasticity. J. Appl. Mech. 50, 554-560.

Lubarda, V.A., Sumarac, D., Krajcinovic, D., 1993. Preisach model and hysteretic behaviour of ductile materials. Eur. J. Mech. A/Solids 12, 445-470.

Malvern, L.E., 1951. The propagation of longitudinal waves of plastic deformation in a bar of material exhibiting a strain-rate effect. J. Appl. Mech. 18, 203-208.

McDowell, D.L., 1992. A nonlinear kinematic hardening theory for cyclic thermoplasticity and thermoviscoplasticity. Int. J. Plasticity 8, 695-728.

Miller, A.K., 1987. Unified Constitutive Equations for Creep and Plasticity. Elsevier Applied Science, London.

Moosbrugger, J.C., McDowell, D.L., 1989. On a class of kinematic hardening rules for nonproportional cyclic plasticity. J. Eng. Mater. Technol. 111, 87-98.

Perzyna, P., 1963. The constitutive equations for rate sensitive plastic materials. Q. Appl. Math 20, 321332.

Perzyna, P., 1966. Fundamental problems in viscoplasticity. Adv. Appl. Mech 9, 243-377.

Ponter, A.R.S., Leckie, F.A., 1976. Constitutive relationships for the time-dependent deformation of metals. J. Eng. Mater. Technol 98, 47-51.

Prager, W., 1956. A new method of analyzing stresses and strains in work-hardening plastic solids. J. Appl. Mech 23, 493-496.

Rice, J.R., 1970. On the structure of stress-strain relations for time-dependent plastic deformation in metals. J. Appl. Mech 37, 728-737.

Rice, J.R., 1971. Inelastic constitutive relations for solids: an internal variable theory and its application to metal plasticity. J. Mech. Phys. Solids 19, 433-455.

Sokolovskii, V.V., 1948. Propagation of elastic-viscoplastic waves in bars. Prikl. Mat. Mekh. 12, 261-280. (in Russian)..

Van Houtte, P., 1994. Application of plastic potentials to strain rate sensitive and insensitive anisotropic materials. Int. J. Plasticity 10, 719-748. 